\title{
Quasi-particle and matrix models of the semi Quark Gluon Plasma
}

\author{
Robert D. Pisarski ${ }^{\mathrm{a}, \mathrm{b}, 1}$, Koji Kashiwa ${ }^{\mathrm{b}}$, Vladimir Skokov ${ }^{\mathrm{a}}$ \\ ${ }^{a}$ Department of Physics, Brookhaven National Laboratory, Upton, NY, 11973 USA \\ ${ }^{b}$ RIKEN/BNL, Brookhaven National Laboratory, Upton, NY, 11973 USA
}

\begin{abstract}
We make a simple observation about two models used to treat the region near the critical temperature of QCD, quasiparticle and matrix models. While they appear very different, we show how these two models might be related. We also present results for the temperature dependence of the ratio of the shear viscosity to the entropy in a matrix model, and suggest that quasi-particle models may behave similarly.
\end{abstract}

\section{Introduction}

The region near the critical temperature in QCD, which can be termed the semi Quark Gluon Plasma, is of fundamental interest. Amongst others, one model used to study the semi-QGP is based upon quasi-particles [1-4]; another, on matrix models [5-13]. While these two approaches appear to have nothing in common, in this note we suggest how they might be related. We then present a result for the temperature dependence of the ratio of the shear viscosity to the entropy in a matrix model.

A quasi-particle model is based upon assuming that the pressure is given by that of an ideal gas of massive gluons. First one computes the pressure for such an ideal gas. Using lattice results for the pressure, one then determines the gluon mass. That is, one trades one function of temperature, namely the pressure, for another, here the gluon mass.

In Fig. (1) we show the behavior of the gluon mass in the pure glue theory with three colors from Ref. [4], but it is very similar to that from Refs. [1-3]. There are two regimes: for temperatures above $\sim 1.2 T_{c}$, the gluon mass is approximately linear in temperature, $m_{g l} \sim T$. This is like the usual Debye mass generated in perturbation theory. In fact, its not quite the same, since in quasi-particle models $m_{g l}$ is a mass for transverse excitations. Still, it is a useful way of thinking about this gluon mass.

Below $\sim 1.2 T_{c}$, the gluon mass increases strongly, as a (fractional) power of $1 /\left(T-T_{c}\right.$ ). This sharp increase in the gluon mass near $T_{c}$ follows inexorably from the assumptions of the model. The lattice data indicates that the pressure of the confined phase is very small [14]. The only parameter to change in a quasi-particle model is the mass, and so for an ideal gas of massive particles to give a small pressure, they must become very heavy, with the pressure strongly suppressed by Boltzmann factors.

One thing of note, which is not usually discussed in quasi-particle models, is clear from Fig. (11). Above $1.2 T_{c}$, there is an an "ordinary" quasi-particle regime, where $m_{g l} \sim T$. In contrast,

\footnotetext{
${ }^{1}$ Talk presented by R. D. P. 


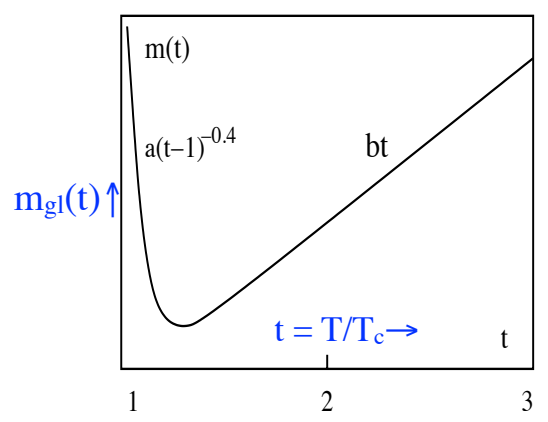

Figure 1: The quasi-particle gluon mass from Ref. [4], for the pure glue theory with three colors.

below $1.2 T_{c}$, there is a "transition" regime, where $m_{g l}$ increases sharply, driving the transition to confinement.

Matrix models appear to be very different from quasi-particle models. One expands about a constant field for the time-like component of the vector potential, $A_{0}$. This is a matrix, albeit extremely simple: it is diagonal, and constant in space-time. There are both perturbative terms in $A_{0}$, and non-perturbative terms, added by hand to drive the transition to confinement [6, 10,-13].

In the matrix model, the non-perturbative terms are $\sim T^{2}$, versus the usual $T^{4}$ terms in perturbation theory. Instead of fitting to the pressure, one fits to the interaction measure, $(e-3 p) / T^{4}$, where $e$ is the energy density. In the pure glue theory, this quantity has a narrow peak below $\sim 1.2 T_{c}$. The parameters of the matrix model are then determined by fitting to this narrow peak. Not surprisingly, the details of the matrix model only matter below $\sim 1.2 T_{c}$. Thus there is a clear division between the region about $\sim 1.2 T_{c}$, and that below. This is similar to the two regimes in the quasi-particle model, as seen in Fig. (1). After discussing some results and limits in the matrix model, we return to this similarity below, in Eq. (1).

The matrix model was developed in the pure glue theory. One clean way of testing the addition of quarks is to use heavy quarks. In the matrix model, the position of the deconfining critical endpoint was computed using the matrix model [12]. This occurs for a heavy quark mass, about twice that for the charm quark, $\sim 2.4 \mathrm{GeV}$ for three degenerate flavors. This result agrees well with recent results in a hopping parameter expansion [15].

We now describe some simple computations in a matrix model in the limit of a large number of colors, $N_{c}$. As for heavy quarks, we add $N_{f}$ flavors of massless quarks, simply by adding the quark contribution to the $A_{0}$ potential perturbatively. We assume that $N_{c}$ is large because then results for the shear viscosity can be taken directly [8, 9]. We give results both for the pure glue theory, $N_{c}=\infty$ and $N_{f}=0$, and for $N_{f}=N_{c}=\infty$.

We compute under the assumption of a uniform eigenvalue density [11]. This is useful because then the model can be solved trivially. The exact solution at infinite $N_{c}$ shows that the eigenvalue density is not constant at $N_{c}=\infty$ [13]. The exact solution exhibits unusual behavior, but we do not expect this to affect $\eta / s$ significantly, though.

In the left panel of Fig. (2) we show the Polyakov loop, $\langle$ loop $\rangle=1 / N_{c} \operatorname{tr} \mathbf{L}$, computed for the pure glue theory, and with dynamical quarks. (Here $\mathbf{L}$ is the thermal Wilson line.) The transition temperature for the pure glue theory is taken to be as for three colors, $T_{d} \sim 270 \mathrm{MeV}$. With massless, dynamical quarks, we assume there is only a chiral transition, at $T_{\chi}$. We take 

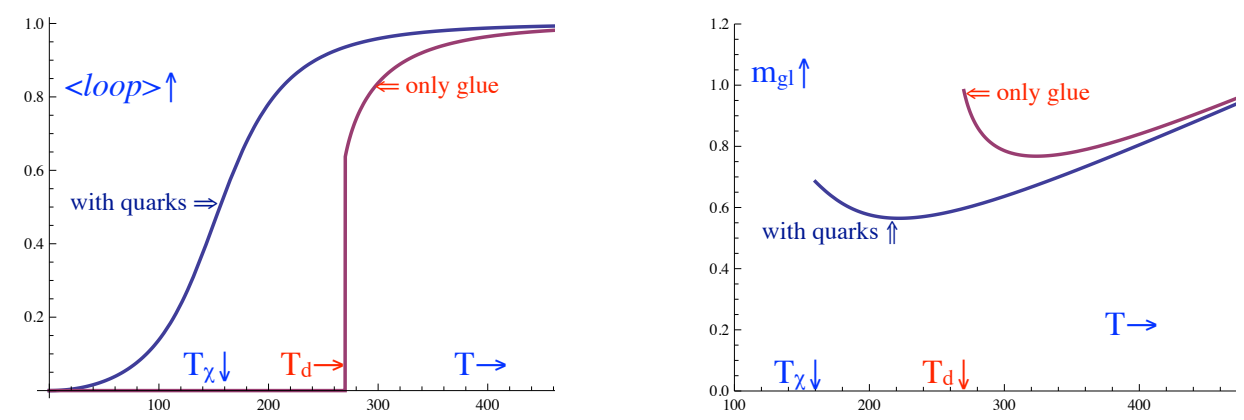

Figure 2: Results from matrix models: expectation values of Polyakov loops (left) and effective gluon masses (right), from Eq. 11. Each graph includes results for the pure glue theory, $N_{f}=0$ and $N_{c}=\infty$, and with dynamical quarks, $N_{f}=N_{c}=\infty$. In the right panel, in the pure glue theory $m_{g l}$ is infinite below $T_{d}$; with quarks, we choose only to show it above $T_{\chi}$, but it continues to increase as the temperature decreases.

$T_{\chi} \sim 160 \mathrm{MeV}$ from three colors. As shown in the left panel, in the pure gauge theory the loop is zero below $T_{d}$, while with dynamical quarks, its expectation value is nonzero at any nonzero temperature. This figure is very similar to results obtained on the lattice for three colors: see, e.g., Fig. 2 of Ref. [14].

We stress that in the matrix model, in principle there is no strong relation between $T_{\chi}$ and $T_{d}$. For a small number of flavors, $N_{f} \ll N_{c}$, then $T_{\chi} \geq T_{d}$, but when $N_{f} \sim N_{c}$, the two are not correlated, and indeed, $T_{\chi}$ can be significantly less than $T_{d}$. Physically, this is because as for three colors, many light quarks simply wash out the deconfining transition.

We now return to the possible similarity between quasi-particle and matrix models. As noted, in both models there is a difference between temperatures above $\sim 1.2 T_{c}$, and those below. Let us try to make this more precise. The Polyakov loop is the propagator for an infinitely heavy quark. To represent how it changes, we define a quasi-particle mass from the expectation value of the loop,

$$
\frac{1}{N_{c}} \operatorname{tr} \mathbf{L}=\mathrm{e}^{-m_{g l} /(\kappa T)}, \kappa=1.7 .
$$

We stress that this definition of the gluon mass is ad hoc. However, it is not completely unreasonable. In the confined phase of a pure gauge theory, the loop vanishes, and so the gluon mass $m_{g l}$ must be infinite. The is like the large (but finite) gluon mass in quasi-particle models. Let is ignore these minor differences, and concentrate on how the gluon mass behaves as one approaches the critical temperature from above.

This leads to our basic point. If we simply take Eq. (1) as given, and use it to compute the gluon mass from the left panel of Fig. (2), then the gluon masses which results, in the left panel of Fig. (2), looks rather like those of the quasi-particle model, in Fig. (1)! Admittedly, this agreement depends sensitively upon the value chosen for the constant $\kappa$ in Eq. (1). Nevertheless, given the definition of the gluon mass, the agreement isn't that surprising. As the loop decreases, the gluon mass must increase.

The agreement is only qualitative. Since in the quasi-particle model the only thing to suppress the pressure is the gluon mass, its increase below $1.2 T_{c}$, Fig. 1, is much sharper than in the matrix model. It is amusing that in the matrix model, the gluon mass appears to be linear in temperature above 1.2 $T_{c}$. This reflects the narrow transition region in the matrix model, so the loop is nearly 
constant.

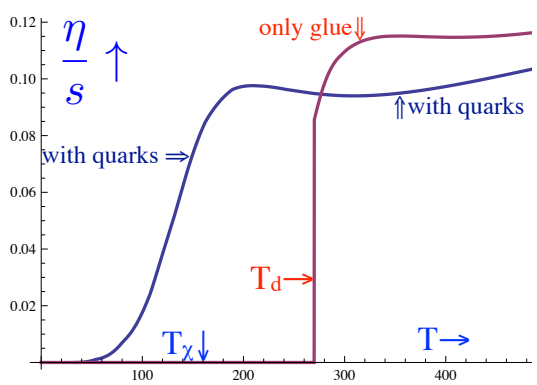

Figure 3: The ratio of the shear viscosity to the entropy from matrix models. Results both with only gluons, with $N_{c}=\infty$, and with dynamical quarks, with $N_{f}=N_{c}=\infty$, are shown.

To illustrate what can be done with the matrix model, in Fig. (3) we show the behavior of the ratio of the shear viscosity to the entropy in the matrix model, following Ref. [9]. This computation was done in the limit of infinite $N_{c}$, which is why we limited ourselves to this case.

In this computation, various constants have been chosen so that the ratio $=1 /(4 \pi)$ at the transition temperature. This is not a natural choice, but made out of convenience. The point of the exercise, which is meant only as illustrative, is to show that while $\eta / s$ is certainly temperature dependent, this can be rather moderate. By the arguments above, we suggest that $\eta / s$ may behave similarly in quasi-particle models.

\section{References}

[1] A. Peshier, B. Kampfer, O. Pavlenko, G. Soff, A Massive quasiparticle model of the SU(3) gluon plasma, Phys.Rev. D54 (1996) 2399-2402.

[2] A. Peshier, W. Cassing, The Hot non-perturbative gluon plasma is an almost ideal colored liquid, Phys.Rev.Lett. 94 (2005) 172301

[3] V. Ozvenchuk, O. Linnyk, M. Gorenstein, E. Bratkovskaya, W. Cassing, Dynamical equilibration of stronglyinteracting 'infinite' parton matter within the Parton-Hadron-String Dynamics (PHSD) transport approach (2012).

[4] P. Castorina, D. E. Miller, H. Satz, Trace Anomaly and Quasi-Particles in Finite Temperature SU(N) Gauge Theory, Eur.Phys.J. C71 (2011) 1673.

[5] R. D. Pisarski, Quark gluon plasma as a condensate of SU(3) Wilson lines, Phys.Rev. D62 (2000) 111501.

[6] P. N. Meisinger, T. R. Miller, M. C. Ogilvie, Phenomenological equations of state for the quark gluon plasma, Phys.Rev. D65 (2002) 034009.

[7] A. Dumitru, Y. Hatta, J. Lenaghan, K. Orginos, R. D. Pisarski, Deconfining phase transition as a matrix model of renormalized Polyakov loops, Phys.Rev. D70 (2004) 034511.

[8] Y. Hidaka, R. D. Pisarski, Suppression of the Shear Viscosity in a "semi" Quark Gluon Plasma, Phys.Rev. D78 (2008) 071501

[9] Y. Hidaka, R. D. Pisarski, Small shear viscosity in the semi quark gluon plasma, Phys.Rev. D81 (2010) 076002

[10] A. Dumitru, Y. Guo, Y. Hidaka, C. P. K. Altes, R. D. Pisarski, How Wide is the Transition to Deconfinement? Phys.Rev. D83 (2011) 034022.

[11] A. Dumitru, Y. Guo, Y. Hidaka, C. P. Korthals Altes, R. D. Pisarski, Effective matrix model for deconfinement in pure gauge theories, Phys.Rev. D (2012).

[12] K. Kashiwa, R. D. Pisarski, V. V. Skokov, Critical endpoint for deconfinement in matrix and other effective models, Phys.Rev. D85 (2012) 114029

[13] R. D. Pisarski, V. V. Skokov, Gross-Witten transition in a matrix model of deconfinement, Phys.Rev. D86 (2012) 081701(R). 
[14] P. Petreczky, Lattice QCD at non-zero temperature, J.Phys. G39 (2012) 093002.

[15] M. Fromm, J. Langelage, S. Lottini, O. Philipsen, The QCD deconfinement transition for heavy quarks and all baryon chemical potentials, JHEP 1201 (2012) 042. 